\title{
Cisto maxilar pós-operatório: relato de caso
}

\author{
Postoperative maxillary cyst: case report \\ Cisto maxilar poste operatório: reporte de caso \\ Mateus Diego PAVELSKI ${ }^{1}$ \\ Maicon Douglas PAVELSKI ${ }^{2}$ \\ Natasha Magro ÉRNICA ${ }^{3}$ \\ Ricardo Augusto $\mathbf{C O N C I}^{3}$ \\ Eleonor Álvaro GARBIN JUNIOR ${ }^{3}$ \\ Geraldo Luiz GRIZA ${ }^{4}$
}

${ }^{I}$ Residente do Serviço de Cirurgia e Traumatologia Bucomaxilofacial da UNIOESTE - Univ. Est. do Oeste do Paraná - CEP 85819-110 - Cascavel - PR, Brasil

${ }^{2}$ Especialista em Cirurgia e Traumatologia Bucomaxilofacial - Univ. do Oeste de Santa Catarina - UNOESC - 89600-000 - Joaçaba - SC, Brasil

${ }^{3}$ Doutor em Cirurgia e Traumatologia Bucomaxilofacial - UNIOESTE - Cascavel - PR, Brasil

${ }^{4}$ Doutor em Implantodontia e professor da UNIOESTE - Cascavel-PR, Brasil

\section{Resumo}

Introdução: Cisto cirúrgico ciliado ou cisto maxilar pós-operatório é uma lesão que ocorre próximo ao ápice dos dentes, porém é originária do epitélio do seio maxilar. É definido como um sequestro da membrana do seio que fica aprisionado e se prolifera, gerando uma cavidade cística verdadeira. Tem aspecto radiográfico semelhante ao cisto radicular, sendo unilocular de centro radiolúcido e bordas radiopacas bem delimitadas. O tratamento é a remoção do epitélio, sendo recomendada a enucleação cirúrgica do cisto. Raramente é relatado recorrências da lesão. Objetivo: relatar um caso clínico e a conduta adotada para resolução do caso de cisto maxilar pós-operatório. Relato de Caso: A paciente de gênero feminino, 59 anos, leucoderma, edentada total, procurou a clínica de odontologia da Universidade Estadual do Oeste do Paraná para confecção de novas próteses totais superiores e inferiores. No exame radiográfico panorâmico, constatou-se uma lesão unilocular em maxila direita. Clinicamente não apresentava nenhuma alteração e nenhuma sintomatologia. Foi realizada a punção da lesão, sob anestesia local, que revelou conteúdo seroso. Em seguida, a lesão foi enucleada. Não houve comunicação com o seio maxilar. A paciente permaneceu em acompanhamento clínico e radiográfico por 3 anos apresentando reparação total da loja sem sinais de recidiva. Conclusão: O acompanhamento à longo prazo é essencial em casos de lesões intraósseas. Mesmo pacientes edêntulos necessitam de exames de imagens para avaliação inicial, descartando alterações intraósseas.

Descritores: Cirurgia Bucal; Cistos Ósseos; Seio Maxilar.

\section{Abstract}

Introduction: Ciliated surgical cyst or postoperative maxillary cyst is an injury that occurs near the apex of the teeth, but originates from the epithelium of the maxillary sinus. It is defined as a sequestering of the sinus membrane that becomes trapped and proliferates, generating a true cystic cavity. It has radiographic appearance similar to the radicular cyst, being unilocular of radiolucent center and radiopaque edges well delimited. Treatment is the removal of the epithelium, and surgical enucleation of the cyst is recommended. Recurrences of the lesion are rarely reported. Objective: to report a clinical case and the conduct adopted to resolve the case of postoperative maxillary cyst. Case Report: The female patient, 59 years old, leucoderma, totally edentate, sought the dentistry clinic of the State University of the West of Paraná to make new upper and lower dentures. Panoramic radiographic examination revealed a unilocular lesion in the right maxilla. Clinically, there were no changes and no symptoms. Puncture of the lesion was performed under local anesthesia, which revealed serous contents. The lesion was then enucleated. There was no communication with the maxillary sinus. The patient remained in clinical and radiographic follow-up for 3 years presenting total repair of the shop without signs of relapse. Conclusion: Long-term follow-up is essential in cases of intraosseous lesions. Even edentulous patients require imaging for initial evaluation, ruling out intra-osseous changes.

Descriptors: Surgery, Oral; Bone Cysts; Maxillary Sinnus.

\section{Resumen}

Introducción: Cisto quirúrgico ciliado o quiste maxilar postoperatorio es una lesión que ocurre cerca del ápice de los dientes, pero es originaria del epitelio del seno maxilar. Se define como un secuestro de la membrana del seno que queda atrapado y se prolifera, generando una cavidad cística verdadera. Tiene un aspecto radiográfico similar al quiste radicular, siendo unilocular de centro radiolúcido y bordes radiopacas bien delimitadas. El tratamiento es la remoción del epitelio, siendo recomendada la enucleación quirúrgica del quiste. Raramente se informa de recurrencias de la lesión. Objetivo: relatar un caso clínico y la conducta adoptada para resolución del caso de quiste maxilar postoperatorio. El paciente de género femenino, 59 años, leucoderma, desdentada total, buscó la clínica de odontología de la Universidad Estadual del Oeste de Paraná para confección de nuevas prótesis totales superiores e inferiores. En el examen radiográfico panorámico, se constató una lesión unilocular en maxila derecha. Clínicamente no presentaba ninguna alteración y ninguna sintomatología. Se realizó la punción de la lesión, bajo anestesia local, que reveló contenido seroso. A continuación, la lesión fue enucleada. No hubo comunicación con el seno maxilar. La paciente permaneció en seguimiento clínico y radiográfico por 3 años presentando reparación total de la tienda sin signos de recidiva. Conclusión: El seguimiento a largo plazo es esencial en casos de lesiones intra-óseas. Incluso los pacientes desdentados necesitan exámenes de imágenes para la evaluación inicial, descartando alteraciones intra-óseas.

Descriptores: Cirugía Bucal; Quistes Óseos; Seno Maxilar.

\section{INTRODUÇÃO}

O cisto cirúrgico ciliado, cisto maxilar pósoperatório ou cisto ciliado traumático é uma lesão intraóssea que ocorre nos seios maxilares, originada de uma implantação iatrogênica de células epiteliais do trato respiratório em uma localização ectópica. É frequentemente observado após cirurgias envolvendo o seio maxilar, porém, há relatos de acometimento mandibular em casos onde houve cirurgias simultâneas envolvendo ambas as regiões ${ }^{1}$. Estas células proliferam-se gerando uma cavidade revestida por epitélio, ou seja, um cisto verdadeiro, separada anatomicamente do seio maxilar, entretanto, como o cisto possui capacidade proliferativa, em alguns casos, sua expansão pode gerar perfurações do assoalho do seio maxilar².

Não há predileção por gênero e acomete principalmente adultos jovens ${ }^{1}$. Clínica e radiograficamente tem aspecto semelhante ao cisto radicular, podendo apresentar dor a palpação e edema intra e extraoral. Os sinas e sintomas variam de acordo com a área atingida pelo crescimento da lesão. A parestesia raramente é descrita. Radiograficamente 
é unilocular e apresenta bordas escleróticas bem definidas, com o lúmen radiolúcido ${ }^{3}$.

O tratamento é definido de acordo com a extensão e invasão da lesão, sendo descritos a marsupialização associada à posterior enucleação ou enucleação cirúrgica direta ${ }^{1,4,5}$. A recidiva é rara, porém, pode ocorrer se todo o epitélio do cisto não for removido ${ }^{6}$.

O objetivo deste trabalho é descrever um caso de diagnóstico e tratamento de um cisto cirúrgico ciliado, assim como realizar uma revisão da literatura sobre as características, etiopatogenia e técnicas e opções disponíveis para o manejo do caso.

\section{CASO CLÍNICO}

Paciente do gênero feminino, 59 anos, procurou a clínica de Odontologia da Universidade Estadual do Oeste do Paraná para confecção de prótese total superior. A tomada radiográfica panorâmica de rotina demonstrou lesão unilocular, hipodensa, de aproximadamente $19 \times 23 \times 11 \mathrm{~mm}$, com bordas escleróticas bem definidas em maxila à direita. A paciente relatou histórico médico de asma e vitiligo, e histórico clínico de exodontia de dentes superiores e inferiores há 39 anos aproximadamente (Figuras 1, 2 e 3 ).

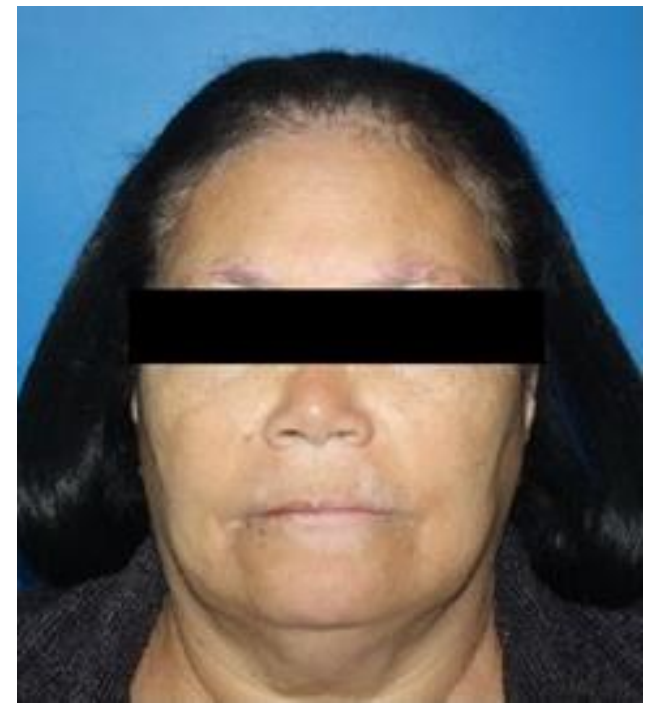

Figura 1: Ausência de assimetria facial.

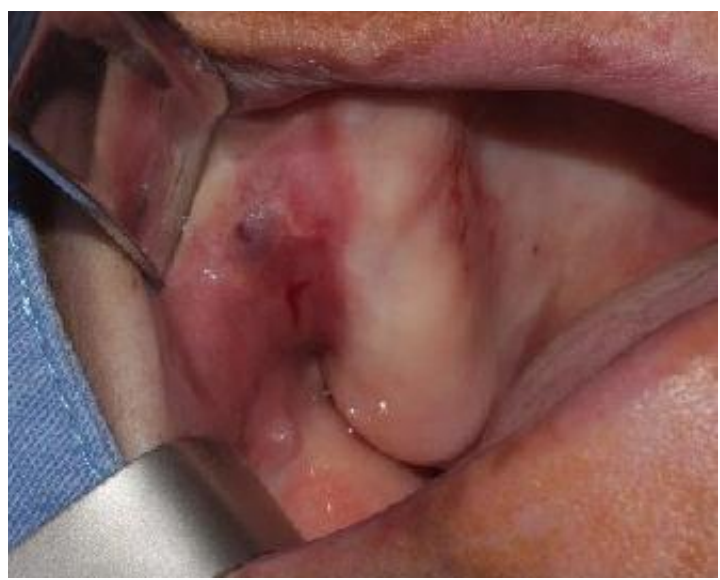

Figura 2: Vista intraoral da maxila direita: ausência de alteração da mucosa bucal.

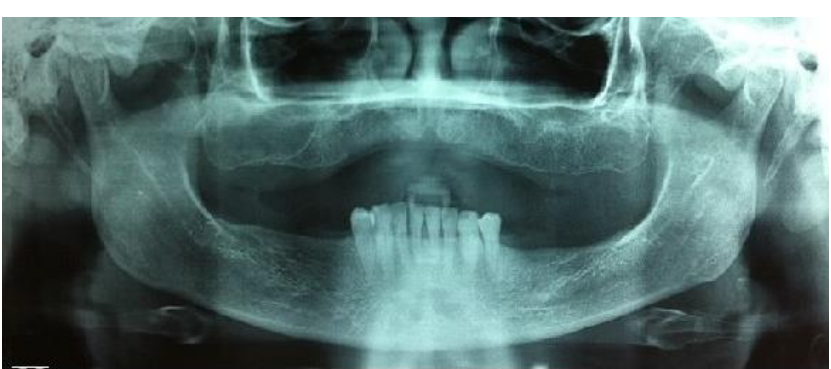

Figura 3: Radiografia panorâmica mostrando lesão em região de maxila direita logo abaixo do seio maxilar.

A enucleação cirúrgica da lesão sob anestesia local foi o tratamento de escolha. Após punção aspirativa, que revelou conteúdo com aspecto seroso e coloração amarelada, foi realizada a incisão monoangular baixa, permitindo o acesso adequado à lesão. Com a elevação mucoperiosteal observou-se fenestração óssea da região, estando a cápsula cística aderida ao periósteo. Ao realizar a divisão dos tecidos, a cápsula cística se rompeu e o líquido marrom-amarelado foi extravasado. A lesão foi enucleada em sua totalidade e a loja óssea irrigada copiosamente com soro fisiológico estéril. Durante a inspeção constatou-se a continuidade óssea, não havendo comunicação com a membrana do seio maxilar. A síntese foi realizada por meio de sutura contínua festonada associada a pontos interrompidos realizados com fio de nylon 5-0, obtendo o fechamento por primeira intenção (Figuras 4, 5, 6 e 7). A mesma foi removida após 10 dias.

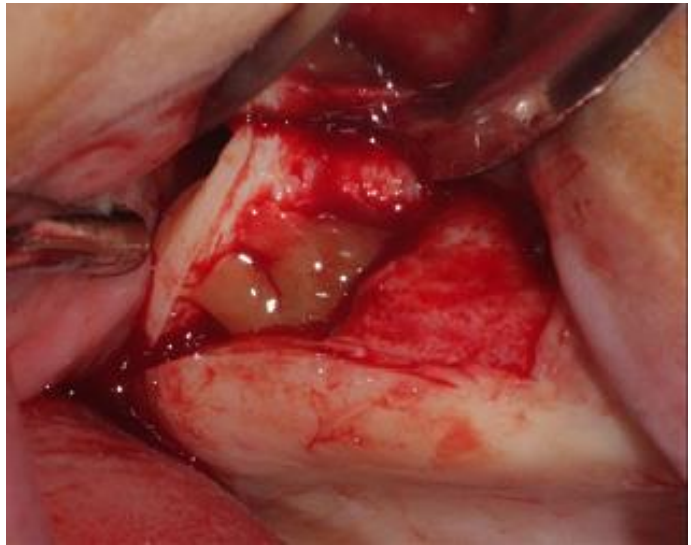

Figura 4: Trans-operatório: fenestração óssea expondo conteúdo cístico.

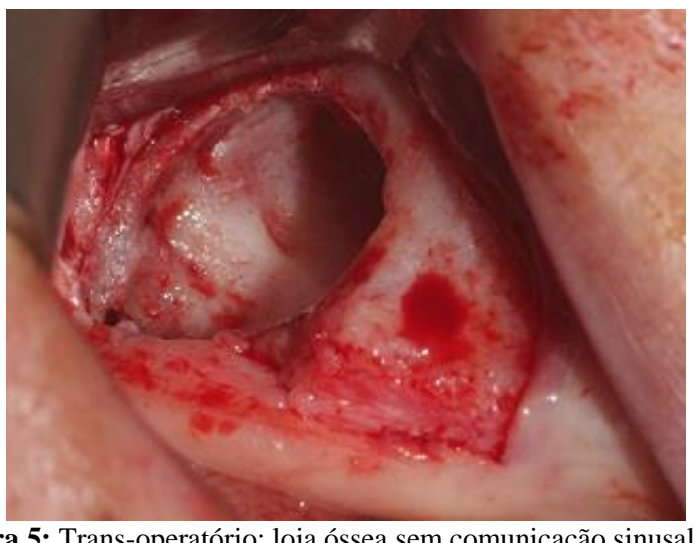

Figura 5: Trans-operatório: loja óssea sem comunicação sinusal. 


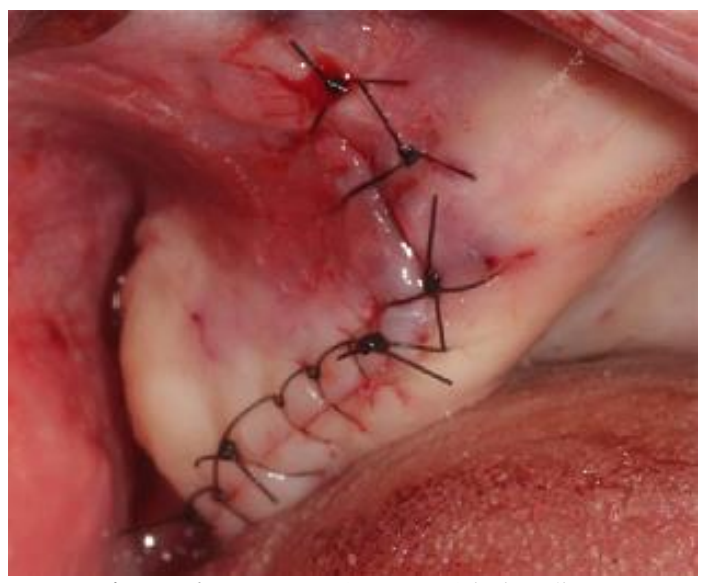

Figura 6: Aspecto pós-operatório imediato.

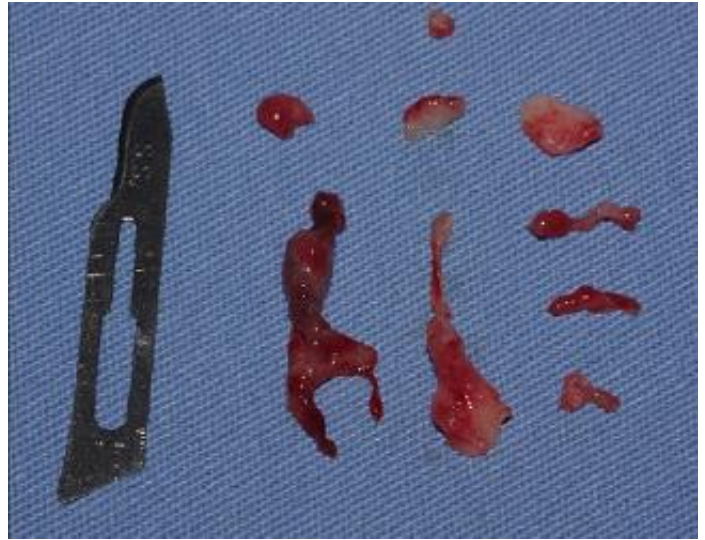

Figura 7: Material coletado enviado para exame histopatológico.

O material coletado foi fixado em solução de formol a $10 \%$ e enviado para o laboratório de patologia da Universidade Estadual do Oeste do Paraná. O laudo histopatológico descreveu epitélio central pseudoestratificado associado a tecido conjuntivo fibroso com um infiltrado inflamatório, e, correlacionando os achados clínicos e histopatológicos. O diagnóstico foi de cisto cirúrgico ciliado. A paciente permaneceu em acompanhamento clínico e radiográfico por 03 anos, sem indícios de recidiva (Figuras 8,9 e 10).

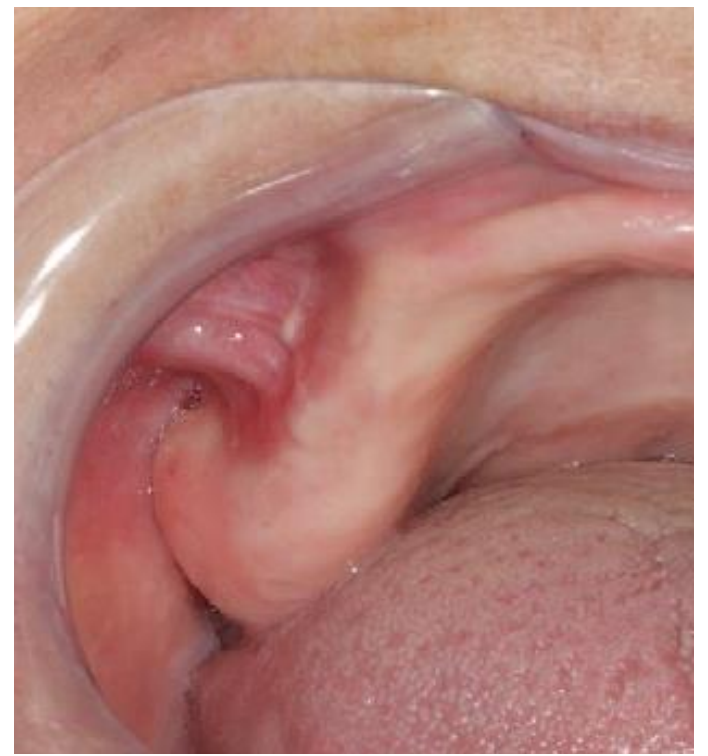

Figura 8: Vista intraoral da maxila direita sem alteração da mucosa bucal.

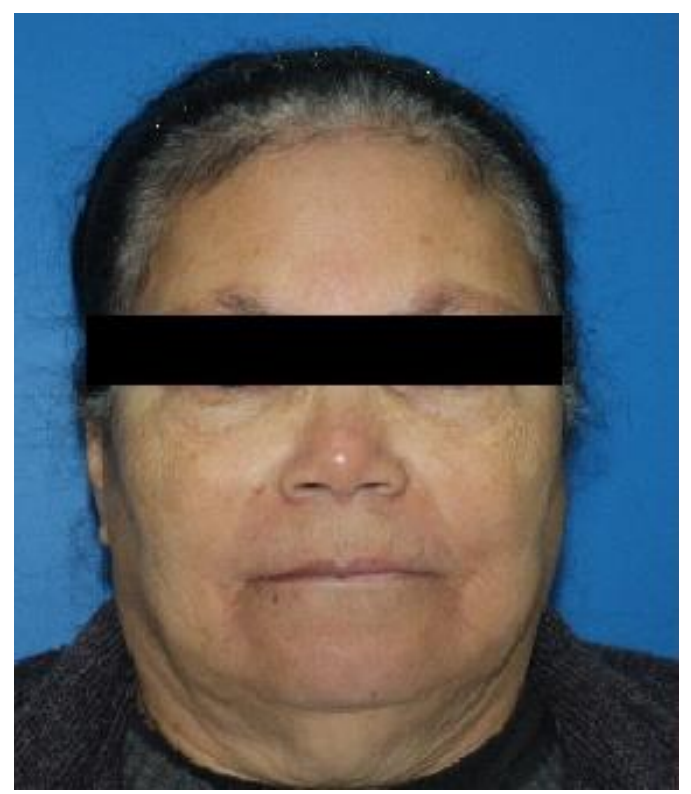

Figura 9: Ausência de assimetria facial.

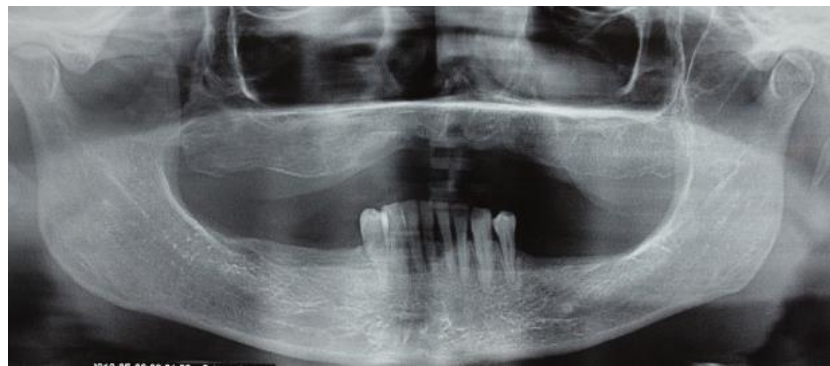

Figura 10: Radiografia panorâmica em pós-operatório de 36 meses mostrando completo reparo ósseo em região de maxila direita.

\section{DISCUSSÃO}

Muito citado na literatura japonesa, o cisto cirúrgico ciliado é uma lesão benigna, localmente agressiva e descrita inicialmente por Kubo, em $1927^{7,8}$. O levantamento bibliográfico utilizando a base de dados Pubmed revela apenas cerca de nove casos nos trabalhos de língua inglesa nos últimos 10 anos. Alguns autores atribuem este fato à alta incidência de sinusite crônica no Japão, ${ }^{9,10}$, especialmente na década de $1970^{11}$. Fernandes et al. ${ }^{8}$ acrescentam ainda $\mathrm{o}$ alto índice de sinusectomias agressivas para o tratamento de sinusite durante a segunda guerra mundial, visto a escassez antibiótica no período. Já Basu et al. ${ }^{12}$ atribuem esta discrepância a outros fatores, como discordâncias no diagnóstico.

Os cistos de implantação ocorrem normalmente por traumas locais, como cirurgias para enxertia óssea ou outros procedimentos com acesso direto ao interior do seio maxilar. Também é descrito após exodontias cujo ápice radicular ou a lesão periápice esteja em intimo contato com a membrana sinusal ${ }^{13-15}$. Outros autores como $\mathrm{Li}$ et al. ${ }^{1}$ afirmam que cirurgias que envolvem simultaneamente o terço médio e inferior de face são a etiologia responsável pelo desenvolvimento destas entidades na região mandibular, sítio distante de seu local nativo. Tal fato está atribuído à contaminação de outros sítios cirúrgicos com células do trato respiratório. 
Após o aprisionamento da mucosa respiratória, citocinas provenientes de um processo inflamatório induziriam a transformação cística das células do epitélio ciliar e, por diferença osmótica, leva à expansão da lesão, que, em alguns casos, pode atingir grandes proporções e causar destruição das estruturas adjacentes. Esta transformação ocorre de maneira tardia, meses a anos após o trauma inicial ${ }^{11}$, com média de desenvolvimento de 10 a 20 anos após a implantação ${ }^{8,16}$. Para $\mathrm{Li}$ et al. ${ }^{1}$ até $20 \%$ dos pacientes diagnosticados com o cisto maxilar pósoperatório desenvolveram a lesão entre 10 e 29 anos após o trauma cirúrgico. No caso em questão, o tempo decorrido para o diagnóstico foi superior ao citado, uma vez que a paciente procurou atendimento 39 anos após a exodontia dos dentes superiores. Por outro lado, existem casos publicados na literatura mundial de cistos descobertos 49 anos após procedimentos cirúrgicos ${ }^{17}$.

No que tange à etiologia, os cistos maxilares pós-operatórios são relatados após cirurgias invasivas no seio maxilar por acesso de Caldwell-Luc, osteotomias do terço médio da face, traumas faciais, ferimentos por projétil de arma de fogo e cirurgias parendodônticas $^{7,18-21}$. Para Fernandes et al. ${ }^{8}$ até 20\% dos pacientes submetidos a estas modalidades cirúrgicas podem desenvolver este tipo de patologia. Contrariando estes achados, a paciente citada não apresenta histórico de qualquer acesso direto ao seio maxilar, tendo realizado apenas as exodontias dos dentes superiores concordando com Marano et al. ${ }^{15}$.

Os relatos de desenvolvimento de cistos de implantação após osteotomias dos maxilares são comuns, como descrito por Moe et al. ${ }^{11}$, Amin et al. ${ }^{16}$, Sugar et al. ${ }^{18}$ e Hayhurst et al. ${ }^{19}$. Porém, de modo menos prevalente, a manipulação bimaxilar no mesmo tempo cirúrgico pode gerar cistos cirúrgicos ciliados distantes da região sinusal. Li et al. ${ }^{1}$ e Bourgeois et al. ${ }^{22}$ relatam cistos na região mandibular após a realização de cirurgia ortognática. Já Jingang et al. ${ }^{23}$ descreveram um cisto pós-operatório na região cantal medial 10 anos após a osteossíntese de fraturas do terço médio de face. Neste caso, a hipótese dos autores seria o desenvolvimento a partir de remanescentes da mucosa do seio etmoidal aprisionados. Há consenso entre os autores que para prevenção destas lesões, as lacerações das mucosas sinusais devem ser suturadas e as feridas ser lavadas e irrigadas de maneira copiosa antes do fechamento, assim como deve ocorrer a limpeza meticulosa das serras e brocas que serão reutilizadas em outros sítios $^{22,23}$. Moe et al. ${ }^{11}$ defendem que, nestes casos, um segundo instrumental deve ser utilizado para evitar os riscos de transferência de células do trato respiratório.

Segundo Amin et al. ${ }^{16}$ e Bourgeois e Nelson $^{22}$, a principal faixa etária é de 28 à 59 anos de idade, com baixo índice de recorrência. Os autores acrescentam ainda que apenas 3,4\% dos pacientes relatam ter história de cirurgias em seio maxilar. Corroborando com estas informações, no presente trabalho, a paciente se encontra dentro da faixa etária descrita na literatura, sem reincidência até o momento e sem histórico de cirurgias diretamente relacionadas ao seio maxilar.

Não há consenso no que diz respeito à predileção por gênero ${ }^{11}$. Li et al. ${ }^{1}$ afirmam não haver predileção por gênero. Por outro lado, Kaneshiro et al. ${ }^{9}$ defendem que há maior predileção por homens, já para Basu et al. ${ }^{12}$ ocorrem mais casos em pacientes do gênero feminino.

O cisto cirúrgico ciliado é mais prevalente na região posterior de maxila, podendo ser uni ou bilateral. Caracteristicamente, apresenta-se como um abaulamento doloroso do processo alveolar e palato, associado a fistula e drenagem ${ }^{8,11 .}$ Já para Li et al. ${ }^{1}$, dor e secreção purulenta estarão presentes apenas se houver infecção secundária. Leung et al. $^{6}$ afirmam que nestes casos, o quadro clínico se assemelha ao de um cisto odontogênico inflamatório. Para estes autores a expansão do cisto pode afetar o fornecimento neurovascular dos dentes adjacentes, podendo estar associado, inclusive, a dentes nãovitais. Basu et al. ${ }^{12}$ avaliaram 23 pacientes quanto a sintomatologia dolorosa, dos quais 22 casos apresentaram sintomatologia dolorosa e somente um mostrou-se assintomático. Contrariando a maioria, a paciente não apresentou qualquer sintomatologia dolorosa em relação à lesão.

Radiograficamente a lesão se apresenta como uma imagem hipodensa, unilocular, bem delimitada por bordas escleróticas, geralmente em íntimo contato com o seio maxilar ${ }^{1,4,22,23}$, de forma semelhante ao caso apresentado. Moe et al. ${ }^{11}$, entretanto, cita que, apesar de menos comum, os cistos podem ser multiloculares ou apresentar continuidade com o seio maxilar.

Devido às características genéricas, $\mathrm{Li}$ et al. ${ }^{1}$ e Fernandes et al. ${ }^{8}$ concordam que muitos cistos e tumores odontogênicos ou não-odontogênicos podem ser considerados em seu diagnóstico diferencial, sendo os principais o cisto odontogênico glandular, cisto ósseo simples, queratocisto e cisto residual. Li et al. ${ }^{1}$ afirmam ainda que muitas vezes estas lesões são diagnosticadas como pseudocistos antrais, não sendo tratadas de maneira adequada. Desta forma, todos os autores consultados condescendem que a associação das características clínicas, radiográficas, histológicas e o histórico clínico de cirurgia prévia envolvendo os seios paranasais são fundamentais para o correto diagnóstico e condução do caso $^{1,8,11,22,23}$.

Assim, quanto ao aspecto microscópico, observa-se uma parede de tecido conjuntivo, que pode conter células inflamatórias, cristais de colesterol e fragmentos de osso reacional. Por vezes, 
uma camada acelular e hialinizada podem ser visualizadas subjacentes ao revestimento epitelial, constituído por epitélio colunar pseudoestratificado ciliado ${ }^{1,10,11}$. Moe et al. ${ }^{11}$ afirmam que áreas de áreas de epitélio escamoso são comuns e, em alguns casos, nenhum epitélio é identificado. Fernandes et al. ${ }^{8}$ complementam que uma variação no epitélio pode ocorrer como resultado de estímulos, como a inflamação crônica, gerando uma metaplasia do epitélio.

Em relação ao tratamento de escolha, a maioria dos autores concordam que a enucleação é mais indicada ${ }^{2,4,5,10,22,23}$. Já Moe et al. ${ }^{11}$ acrescentam que a enucleação deve ser associada à curetagem vigorosa da loja cirúrgica para remoção de qualquer remanescente cístico alojado nos contornos da lesão. Kim et al. ${ }^{7}$ e $\mathrm{Pe}$ et al. ${ }^{25}$ afirmam que a marsupialização deve ser indicada em lesões muito extensas, ou quando a abordagem intraoral não for possível, na tentativa de evitar uma abordagem extra oral. Yoshikawa et al. ${ }^{4}$ e Kaneshiro et al. ${ }^{9}$ asseguram que tanto a marsupialização quanto a enucleação são tratamentos efetivos para o cisto cirúrgico ciliado, ficando a critério do cirurgião a conduta a ser adotada.

Lee e Lee ${ }^{26}$ realizaram uma pesquisa com 60 pacientes com diagnóstico de cisto maxilar pósoperatório, revelando uma taxa de recidiva de $20 \%$ em tempo médio de 4,7 anos, após a cirurgia. Para Moe et al. ${ }^{11}$ os casos de recorrência são relacionados à remoção incompleta da lesão.

\section{CONSIDERAÇÕES FINAIS}

O cisto maxilar pós-operatório, apesar de incomum, deve ser considerado como diagnóstico diferencial em lesões com histórico de cirurgias prévias, sejam elas médicas ou odontológicas.

Como conclusão exalta-se importância da avaliação de exames por imagem, sendo indispensável para detectar lesões intraósseas independentemente da queixa principal relatada.

Vale ressaltar ainda que o acompanhamento em longo prazo de lesões intraósseas é essencial para o sucesso do tratamento e para detecção inicial de possíveis reincidências de lesões.

\section{REFERÊNCIAS}

1. Li CC, Feinerman DM, MacCarthy KD, Woo SB. Rare mandibular surgical ciliated cysts: report of two new cases. J Oral Maxillofac Surg. 2014;72(9):1736-43.

2. Neville BW, Damm DD, Allen CM, Bouquot JE. Patologia oral e maxilofacial. 3. ed. Rio de Janeiro: Elsevier; 2009.

3. Shafer WG, Hine MK, Levy BM, Tomich CE. Tratado de Patologia Bucal. 4. ed. Rio de Janeiro: Interamericana; 1985.

4. Yoshikawa Y, Nakajima T, Kaneshiro S, Sakaguchi M. Effective treatment of the postoperative maxillary cyst by marsupialization. J Oral Maxillofac Surg. 1982;40(8):487-91.

5. Thio D, De S, Phelps PD, Bath AP. Maxillary sinus mucocele presenting as a late complication of a maxillary advancement procedure. J Laryngol Otol. 2003;117(5):402-3.

6. Leung YY, Wong WY, Cheung LK. Surgical ciliated cysts may mimic radicular cysts or residual cysts of maxilla: report of 3 cases. J Oral Maxillofac Surg. 2012;70(4):e264-69.

7. Kim J, Nam IC, Yun SH, Cho JH. A huge midline premaxillary cyst as a late complication of maxillary surgery. J Craniofac Surg. 2011;22(5):1903-5.

8. Fernandes KS, Gallottini MHC, Felix VB, Santos PSS, Nunes FD. Surgical ciliated cyst of the maxilla after maxillary sinus surgery: a case report. Oral Sur. 2013;6(4):229-33.

9. Kaneshiro S, Nakajima T, Yoshikawa Y, Iwasaki $\mathrm{H}$, Tokiwa N. The postoperative maxillary cyst: report of 71 cases. J Oral Surg. 1981;39(3):191-98.

10.Cano J, Campo J, Alobera MA, Baca R. Surgical ciliated cyst of the maxilla. Clinical case. Med Oral Patol Oral Cir Bucal. 2009;14(7):e361-64.

11. Moe JS, Magliocca KR, Steed MB. Early maxillary surgical ciliated cyst after Le Fort I untreated for 20 years. Oral Surg. 2013;6(4):224-28.

12. Basu MK, Rout PGJ, Rippin JW, Smith AJ. The post-operative maxillary cyst: Experience with 23 cases. Int J Oral Maxillofac Surg. 1988; 17(5):282-84

13. Yamamoto H, Takagi M. Clinicopathologic study of the postoperative maxillary cyst. Oral Surg Oral Med Oral Pathol. 1986;62(5):544-48.

14.Heo MS, Song MY, Lee SS, Choi SC, Park TW. A comparative study of the radiological diagnosis of postoperative maxillary cyst. Dentomaxillofacial Radiol. 2000;29(6):347-51.

15.Marano R, Santos SE, Sawazaki R, de Moraes M. Um raro caso de cisto cirúrgico ciliado após 5 anos de extração dentária. Rev Port Estomatol Med Dent Cir Maxilofac. 2012;53:246-51.

16. Amin M, Witherow H, Lee R, Blenkinsopp P. Surgical ciliated cyst after maxillary orthognathic surgery: report of a case. J Oral Maxillofac Surg. 2003;61(1):138-41.

17. Shik CK. The post-operative maxillary cyst: report of 14 cases. Taehan Chikkwa Uisa Hyophoe Chi. 1989;27(11):1049-57.

18.Sugar AW, Walker DM, Bounds GA. Surgical ciliated (postoperative maxillary) cysts following mid-face osteotomies. Br J Oral Maxillofac Surg. 1990;28(4):264-67.

19. Hayhurst DL, Moenning JE, Summerlin DJ, Bussard DA. Surgical ciliated cyst: a delayed complication in a case of maxillary orthognathic surgery. J Oral Maxillofac Surg. 1993;51(6):705-8.

20.Lockhart R, Ceccaldi J, Bertrand JC. 
Postoperative maxillary cyst following sinus bone graft: report of a case. Int J Oral Maxillofac Implants. 2000;15(4):583-86.

21. Shakib K, McCarthy E, Walker DM, Newman L. Post operative maxillary cyst: report of an unusual presentation. $\mathrm{Br} \mathrm{J}$ Oral Maxillofac Surg. 2009;47(5):419-21.

22. Bourgeois SL Jr, Nelson BL. Surgical ciliated cyst of the mandible secondary to simultaneous Le Fort I osteotomy and genioplasty: report of case and review of the literature. Oral Surg Oral Med Oral Pathol Oral Radiol Endod. 2005;100(1):36-9.

23. An J, Zhang Y. Surgical ciliated cyst of the medial canthal region after the management of a midfacial fracture: a case report. J Craniofac Surg. 2014;25(2):701-2.

24. Samuels HS. Marsupialization: Effective management of large maxillary cysts: report of a case. Oral Surg Oral Med Oral Pathol. 1965;20(5):676-83.

25.Pe MB, Sano K, Kitamura A, Inokuchi T. Computed tomography in the evaluation of postoperative maxillary cysts. J Oral Maxillofac Surg. 1990;48(7):679-84.

26.Lee KC, Lee NH. Comparison of clinical characteristics between primary and secondary paranasal mucoceles. Yonsei Med J. 2010;51(5):735-39.

\section{CONFLITO DE INTERESSES}

Os autores declaram não haver conflitos de interesse.

\section{AUTOR PARA CORRESPONDENCIA}

\section{Mateus Diego Pavelski}

mateus_pavelski@hotmail.com

Submetido em 08/11/2018

Aceito em 12/03/2019 